SCIENTIFIC LETTER

\title{
Adequate intracoronary adenosine doses to achieve maximum hyperaemia in coronary functional studies by pressure derived fractional flow reserve: a dose response study
}

\author{
R Lopez-Palop, D Saura, E Pinar, I Lozano, F Pérez-Lorente, F Picó, M Valdez
}

Heart 2004;90:95-96

C oronary pressure derived fractional flow reserve (FFR) is an increasingly used invasive index of the functional significance of coronary lesions. FFR expresses maximum achievable blood flow to the myocardium, supplied by a stenotic artery, as a fraction of normal maximum flow. ${ }^{12}$ Achievement of maximal hyperaemia is essential for calculation of FFR. Intracoronary adenosine is widely used to obtain maximal hyperaemia. Although standard doses of intracoronary adenosine to achieve maximal hyperaemia have been well established in previous studies ${ }^{3}$ (15-40 $\mu$ g left coronary artery, $10-30 \mu \mathrm{g}$ right coronary artery), doubts about maximal hyperaemia achieved with these doses have led to the empirical use of higher than standard doses in clinical practice. ${ }^{45}$ The aim of this study was to analyse the effects of incremental doses of intracoronary adenosine on FFR measurements.

\section{METHODS}

Patients with angiographically intermediate lesions (visual percentage diameter stenosis $50-75 \%$ ) in a principal coronary artery and indication for study with pressure wire to determine functional significance of lesions were included. The study started on November 2001 and 50 lesions were prospectively and consecutively included. Informed consent was obtained from all patients.

Coronary pressure measurements were performed using a 0.014 inch pressure guidewire (PressureWire, Radi Medical Systems, Uppsala, Sweden), according to a previously described technique. ${ }^{1}$ Special attention was paid to avoiding arterial pressure wave damping, unselective catheterisation of coronary ostia, and variation in the position of the pressure guide wire.

Dose administration protocol was started after an initial intracoronary injection of $200 \mu \mathrm{g}$ of glyceryl trinitrate. Incremental doses of intracoronary adenosine of $0,15,30$, $60,90,120,150,180$, and $210 \mu \mathrm{g}$ were tested. Each adenosine bolus was administered diluted in $5 \mathrm{ml}$ of normal saline and was immediately followed by a $10 \mathrm{ml}$ flush of normal saline as transport medium. Immediately after each dose administration, the $\mathrm{Pd} / \mathrm{Pa}$ ratio was determined from beat-to-beat mean signal. Pd represents the mean coronary pressure distal to the stenotic segment studied measured by pressure wire, and $\mathrm{Pa}$ represents the mean aortic pressure simultaneously measured by the guiding catheter. Each dose was given twice and the $\mathrm{Pd} / \mathrm{Pa}$ ratio value was taken as the average of both measurements. The minimum FFR achieved for each lesion was taken as the nearest approximation to correct FFR (when maximal hyperaemia is obtained) and called true FFR. Comparisons between $\mathrm{Pd} / \mathrm{Pa}$ ratio value observed with standard doses and true FFR obtained in each lesion were obtained.
Differences between groups were tested using Student's $t$ statistic. A probability value of $p<0.05$ was considered significant. SPSS for Windows, version 11.0, was used for the statistical test.

\section{RESULTS}

Sixty lesions in 56 patients were studied. Seventy one per cent of procedures were performed in patients with an acute coronary syndrome. Forty nine (82\%) "de novo" and 18 $(11 \%)$ in-stent restenosis lesions were studied (mean (SD) diameter stenosis $56.11(8.6) \%)$. The vessels studied were anterior descending (67\%), circumflex (15\%), and right (18\%) coronary arteries. Forty three lesions were medically treated $(72 \%)$ and $17(28 \%)$ were revascularised according to FFR results.

Seventeen lesions (23\%) reached a mean value of FFR $<0.75$ with some pairs of doses of adenosine. The mean (SD) of difference between $\mathrm{Pd} / \mathrm{Pa}$ ratio with the $15 \mu \mathrm{g}$ dose and true FFR was 0.04 (0.03).

The true FFR value was observed only in $17 \%$ of lesions with $15 \mu \mathrm{g}$ of adenosine and $23 \%$ with $30 \mu \mathrm{g}$. In $17 \%$ of patients $210 \mu \mathrm{g}$ was necessary to reach the true FFR value. Nine lesions (14\%) that achieved an initial $\mathrm{Pd} / \mathrm{Pa}$ value $>0.75$ with $15 \mu \mathrm{g}$ of intracoronary adenosine had a decrease in $\mathrm{Pd} / \mathrm{Pa}$ value to $<0.75$ with subsequent incremental doses of adenosine. None of 31 lesions with a $\mathrm{Pd} / \mathrm{Pa}$ value $\geqslant 0.90$ with $15 \mu \mathrm{g}$ dose achieved a $\mathrm{Pd} / \mathrm{Pa}$ value $<0.75$ with incremental doses. Nine $(31 \%)$ of 29 lesions with a $\mathrm{Pd} / \mathrm{Pa}$ value between 0.76 and 0.85 with a $15 \mu \mathrm{g}$ dose showed a significant $\mathrm{Pd} / \mathrm{Pa}$ ratio value $<0.75$ with higher doses ( 8 of 9 lesions (89\%) if the $\mathrm{Pd} / \mathrm{Pa}$ ratio value with $15 \mu \mathrm{g}$ was between $>0.75$ and 0.79 ) (fig 1 ).

There were no adverse events related to adenosine administration except for transient asymptomatic atrioventricular blocks immediately after the adenosine bolus was administered.

\section{DISCUSSION}

This study shows that standard doses of intracoronary adenosine do not achieve maximal hyperaemia in the majority of lesions. Although the mean absolute value of difference between low and high doses was small (0.04), this difference was significantly higher (0.07) in lesions with a borderline $(\leqslant 0.80) \mathrm{Pd} / \mathrm{Pa}$ ratio value.

Abbreviations: FFR, fractional flow reserve; $\mathrm{Pa}$, mean aortic pressure simultaneously measured by the guiding catheter; Pd, mean coronary pressure distal to the stenotic segment studied measured by pressure wire 


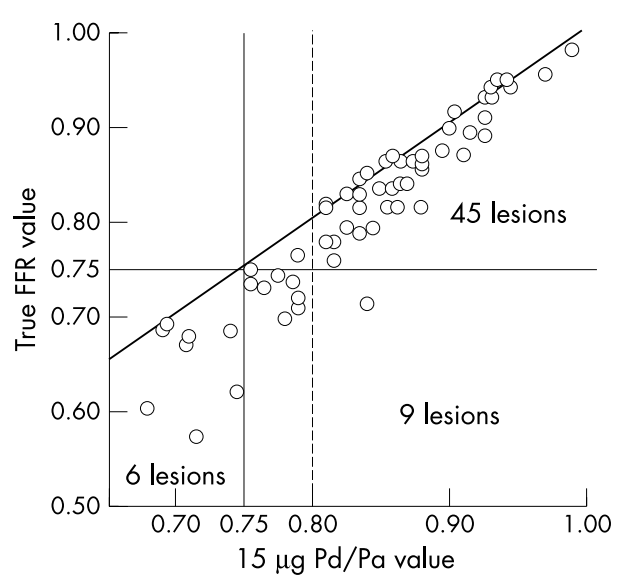

Figure $1 \mathrm{Pd} / \mathrm{Pa}$ values obtained with $15 \mu \mathrm{g}$ dose of intracoronary adenosine versus true fractional flow reserve (FFR) value. Nine lesions with $15 \mu \mathrm{g}$ intracoronary adenosine dose $\mathrm{Pd} / \mathrm{Pa}$ value $>0.75$ reached a significant value $(<0.75$ ) with higher doses (one of them with $15 \mu \mathrm{g}$ intracoronary adenosine dose $\mathrm{Pd} / \mathrm{Pa}$ value $>0.80$ ).

The impaired hyperaemic response caused by microvascular dysfunction in several clinical situations may explain the observed incomplete vasodilatation with the lowest doses of intracoronary adenosine. A damaged microcirculation could respond to high doses but not to low doses of intracoronary adenosine. This "quantitative" response, although theoretically possible, has not been previously demonstrated. On the other hand, first doses would theoretically facilitate the effect of subsequent doses of adenosine, increasing the flow and "effective" local dose of adenosine in the arteriolar region. Finally, in contrast to intravenous administration, the delivery technique of an intracoronary bolus injection is of critical importance. Although experienced interventionists usually take extra care to ensure a correct intracoronary injection, sometimes a backflow into the aorta cannot be avoided and reliable drug administration into the coronary system cannot be warranted.

The results of our study have several clinical implications. Although the average difference observed between FFR obtained with standard and higher doses was small, this difference was higher in lesions with borderline FFR $(0.85-$ 0.75 ), changing the initial functional consideration of these lesions and therefore decisions about their treatment. This study supports the empirical recommendations ${ }^{4}$ and clinical practice $^{5}$ of use of higher doses of intracoronary adenosine, but mainly in lesions with a standard dose FFR value $<0.85$. The use of higher than standard doses in all lesions may not be useful, but the use of $>100 \mu \mathrm{g}$ of intracoronary adenosine or an incremental dose approach if an FFR value $<0.85$ is obtained with standard doses may be clearly recommended.

\section{Authors' affiliations \\ R Lopez-Palop, D Saura, E Pinar, I Lozano, F Pérez-Lorente, F Picó, M Valdez, Department of Cardiology, Hospital Universitario Virgen de la Arrixaca, Murcia, Spain}

Correspondence to: Dr Ramon Lopez-Palop, Ricardo Gil n²0, $3^{\circ}$ Dcha, 30002, Murcia, Spain; mlopezs@meditex.es

Accepted 19 June 2003

\section{REFERENCES}

1 Pijls NH, van Gelder B, Van der Voort P, et al. Fractional flow reserve. A useful index to evaluate the influence of an epicardial coronary stenosis on myocardial blood flow. Circulation 1995:92:3183-93.

2 Pijls NH, De Bruyne B, Peels K, et al. Measurement of fractional flow reserve to assess the functional severity of coronary-artery stenoses. N Engl J Med 1996;334:1703-8.

3 Wilson RF, Wyche K, Christensen BV, et al. Effects of adenosine on human coronary arterial circulation. Circulation 1990;82:1595-606.

4 Pijls NH, Klauss V, Siebert U, et al. Coronary pressure measurement after stenting predicts adverse events at follow-up: a multicenter registry. Circulation 2002; 105:2950-4.

5 Lopez-Palop R, Pinar E, Lozano I, et al. Clinical utilization of the coronary pressure wire. Rev Esp Cardiol 2002;55:251-7.

\section{WEB TOP 10}

www.heartinl.com

These articles scored the most hits on Heart's website during October 2003

1 Left ventricular remodelling after myocardial infarction: importance of residual myocardial viability and ischaemia C A Visser

October 2003;89:1121-2. (Editorial)

2 Basic aspects of plaque vulnerability

A Lafont

October 2003;89:1262-7. (Education in Heart)

3 Antiplatelet treatment in stable coronary artery disease C J Knight

October 2003;89:1273-78. (Education in Heart)

4 Plaque stabilisation in acute coronary syndromes: clinical considerations

A D Timmis

October 2003;89:1268-72. (Education in Heart)

5 Inflammatory and thrombotic mechanisms in coronary atherosclerosis

D Tousoulis, G Davies, C Stefanadis, P Toutouzas, JA Ambrose

September 2003;89:993-7. (Review)

6 Atrial fibrillation: classification, pathophysiology, mechanisms and drug treatment

$\checkmark$ Markides, RJ Schilling

August 2003;89:939-43. (Education in Heart)

7 Joint British recommendations on prevention of coronary heart disease in clinical practice

December 2001, 1998;80:1-29.

8 Influence of contractile reserve and inducible ischaemia on left ventricular remodelling after acute myocardial infarction C Coletta, A Sestili, F Seccareccia, R Rambaldi, R Ricci, A Galati, R Bigi, N Aspromonte, M Renzi, V Ceci

October 2003;89:1138-43. (Cardiovascular medicine)

9 Practical aspects of the management of pericardial disease

B Maisch, A D Ristic

September 2003;89:1096-103. (Education in Heart)

10 Off-pump coronary artery bypass graft surgery: the incidence of postoperative atrial fibrillation

$R$ A Archbold, N P Curzen

October 2003;89:1134-7. (Review)

Visit the Heart website for hyperlinks to these articles, by clicking on "Top 10 papers"

www.heartinl.com 